\title{
(DES)AJUSTAMENTO PSICOLÓGICO E EXPRESSÕES IDIOMÁTICAS NA ADOLESCÊNCIA
}

\author{
Maria João Carapeto \\ Universidade de Évora, Escola de Ciências Sociais \\ Departamento de Psicologia \\ mjoaocarapeto@gmail.com
}

Fecha de Recepción: 19 Mayo 2019

Fecha de Admisión: 25 Septiembre 2019

\begin{abstract}
RESUMO
A adolescência é reconhecidamente um período de espantosos avanços desenvolvimentais mas também de algumas vulnerabilidades. Atentas a tal, as sociedades modernas têm vindo a criar serviços para apoio ao desenvolvimento de trajetórias adaptativas na adolescência. Neste sentido, alguns autores têm chamado a atenção para a necessidade de se estabelecerem pontes entre a linguagem de senso comum usada pelos adolescentes quando se referem aos seus problemas de ajustamento psicológico/psicossocial e a linguagem técnica-científica dos profissionais 'psi'. Neste contexto, os objetivos deste trabalho são os de explorar, em adolescentes e considerando possíveis diferenças de género, a associação entre duas expressões idiomáticas relativas ao ajustamento psicológico comuns em Portugal, "sofrer dos nervos" (SN) e "ter problemas pessoais" (PP), e diversas dimensões de sintomatologia psicológica comuns na literatura científica da saúde mental. Dois instrumentos foram aplicados a uma amostra de alunos do $12^{\circ}$ ano em escolas no sul de Portugal: um questionário de dados pessoais, que incluiu dois itens relativos à autoperceção de SN e de PP, e o Inventário de Sintomas Psicopatológicos. Os resultados sugerem que as autoperceções de SN e de PP estão significativamente correlacionadas, e a correlação é especialmente forte no género feminino. Sugerem ainda que, para ambos os géneros, a correlação mais forte de SN é com ansiedade. Já a autoperceção de PP revela correlações mais fortes com psicoticismo, depressão, ideação paranoide e sensibilidade interpessoal, no género masculino, e, no género feminino, com depressão e ideação paranoide. Finalmente, a ansiedade revelou ser o melhor preditor de SN nos dois géneros, e os melhores preditores de PP são o psicoticismo, nos rapazes, e a ideação paranoide, nas raparigas. Os resultados são discutidos à luz da literatura sobre (des)ajustamento psicológico na adolescência e expressões idiomáticas de desajustamento.

Palavras chave: adolescentes; "sofrer dos nervos"; "ter problemas pessoais"; ansiedade; diferenças de género.
\end{abstract}




\title{
(DES)AJUSTAMENTO PSICOLÓGICO E EXPRESSÕES IDIOMÁTICAS NA ADOLESCÊNCIA
}

\begin{abstract}
Idioms of distress and psychological (mal)adjustment in adolescence. Adolescence is a period of amazing developmental advances but also of some vulnerabilities. With this in mind, modern societies have been creating services to support the development of adaptive pathways through adolescence. Some authors have drawn attention to the need of establishing bridges between the common-sense language used by adolescents to express their psychological/psychosocial adjustment problems and the technical-scientific language of 'psych' professionals. In this context, the objectives of this work are to explore the association between two idioms of distress common in Portugal, "suffering from the nerves" (SN) and "having personal problems" (PP), and several dimensions of psychological symptomatology common in the scientific literature on mental health, in adolescents and accounting for possible gender differences. A sample of 12th grade students in schools of Southern Portugal responded to two instruments: a personal data questionnaire, which included two items related to self-perceptions of SN and PP, and the Brief Symptom Inventory. The results suggest that self-perceptions of SN and PP are significantly correlated and that the correlation is stronger in females. Results also suggest that, for both genders, the SN stronger correlation is with anxiety. Self-perception of PP reveals, on the one hand, stronger correlations with psychoticism, depression, paranoid ideation and interpersonal sensitivity in males and, on the other hand, correlations with depression and paranoid ideation in females. Finally, anxiety proved to be the best predictor of NS in both genders; and the best predictors of PP are psychoticism, in boys, and paranoid ideation, in girls. The results are discussed in light of the literature on psychological (mal)adjustment in adolescence and on idioms of distress.
\end{abstract}

Keywords: adolescents; "suffering from the nerves"; "having personal problems"; anxiety; gender differences.

\section{INTRODUÇÃo}

A adolescência é entendida como um período de muitos ganhos desenvolvimentais ao nível físico, cognitivo, emocional, social, ou do autoconhecimento, entre outros (e.g., Coleman, 2011). No entanto, vários autores têm também chamado a atenção para algumas vulnerabilidades que, embora transitórias na maior parte das vezes, ocasionam desajustamento psicológico e têm potencial para afetar a qualidade das trajetórias desenvolvimentais futuras (Arnett, 1999; Carapeto \& Feixas, 2019; Cicchetti \& Rogosch, 2002). Neste sentido, as sociedades ditas desenvolvidas têm vindo a criar serviços para apoiar o desenvolvimento adaptativo dos adolescentes nas comunidades em que vivem.

Neste contexto, alguns autores têm chamado a atenção para a necessidade de se estabelecerem pontes entre a linguagem de senso comum usada pelas pessoas quando se referem aos seus problemas de ajustamento psicológico/psicossocial e a linguagem técnico-científica dos profissionais "psi" (Abreu, 2007; American Psychiatric Association - APA, 2013; Causadias, 2013; Kaiser, Haroz, Kohrt, Bolton, Bass \& Hinton, 2015).

A psicopatologia cultural (Causadias, 2013), em particular, tem vindo a chamar a atenção para os "conceitos culturais de desajustamento psicológico" (cultural concepts of distress), que consistem nos "modos em que os grupos culturais experienciam, compreendem e comunicam o sofrimento, problemas comportamentais ou pensamentos e emoções perturbadores" (APA, 2013, p. 758). Estas conceções culturais são importantes heurísticas para guiar a atuação das pessoas na sua vida quotidiana e definem que experiências hão de ser assinaladas como desajustamento pelos próprios indivíduos e pelos outros, o que justifica a procura de ajuda, a aderência a intervenções destinadas a tratá-Ios e os respetivos resultados. Os conceitos culturais de desajustamento incluem "síndromas culturais", "modelos explicativos ou causais da perturbação mental", e "expressões 
idiomáticas de desajustamento psicológico". Estas últimas são de especial interesse neste estudo, e podem definir-se como "modos de expressar desajustamento psicológico que podem não envolver sintomas ou síndromes específicos, mas proporcionam modos coletivos, partilhados, de experienciar e falar acerca de preocupações pessoais ou sociais" (APA, 2013, p. 758). Algumas das mais estudados são "sofrer dos nervos" (Alves, 2011; James, Fernandes, Navara, Harris \& Foster, 2009; Nogueira, Mari, \& Razzouk, 2015), "pensar demais" (Kaiser et al., 2015), "cismas" (Alves, 2011), ou "agonias" (James, Slocum \& Zumbo, 2004). Por seu lado, algumas destas expressões idiomáticas têm sido estudadas em diferentes sociedades, como por exemplo "sofrer dos nervos" e "pensar demais".

Em Portugal, não há muita investigação sobre expressões idiomáticas de desajustamento, embora existam alguns estudos com mulheres portuguesas dos Açores imigrantes nos Estados Unidos da América e no Canadá (James et al., 2009; James et al., 2004). No entanto, um estudo qualitativo de Alves (2011) explora, de um ponto de vista mais antropológico ou sociológico, as crenças sobre saúde e doença mental em adultos do norte de Portugal, e dele emergem expressões idiomáticas como sofrer dos nervos, cismas, "doença da cabeça" ou "doença dos nervos".

São escassos os estudos que procuram estabelecer relações sistemáticas entre expressões idiomáticas e categorias de diagnóstico em psicopatologia (e.g., DSM 5, APA, 2013), embora surjam referências gerais a estas relações e sobretudo em adultos (e.g., James et al., 2009).

Neste contexto, este trabalho foca esta relação entre duas expressões idiomáticas de desajustamento psicológico comuns na sociedade portuguesa, sofrer dos nervos e "ter problemas pessoais", e várias expressões técnico-cientificas dos profissionais de saúde mental, que frequentemente se encontram nos sistemas de classificação das perturbações mentais, como a DSM 5 (APA, 2013) ou o ICD 10 (World Health Organization, 1992).

Várias razões justificam a relevância de se estudar as percepções dos adolescentes acerca do seu próprio (des)ajustamento psicológico. Primeiro, apesar de a adolescência ser um período de espantosas oportunidades desenvolvimentais, é também um período de maior risco de desajustamento psicológico (Arnett, 1999; Cichetti \& Rogosch, 2002). A quantidade e a diversidade das transições com que os adolescentes se defrontam desafiam a sua adaptação psicológica e psicossocial, e alguns dos inquestionáveis ganhos desenvolvimentais trazem, apesar disso, novas vulnerabilidades à saúde mental dos adolescentes (Carapeto \& Feixas, 2019).

Segundo, a investigação tem vindo a identificar alguns problemas psicológicos que tendem a emergir ou a ser mais frequentes durante a adolescência, alguns dos quais especialmente internalizadas ou reservados na sua expressão (como é o caso da depressão) (Arnett, 1999; Cicchetti \& Rogosch, 2002; Hankin, 2015), e cujos efeitos podem ser evitados ou minimizados mediante intervenções preventivas (e.g., Garber et al., 2018). As diferenças de género, são aqui assinaláveis, com as raparigas a exibir mais sintomas internalizados e os rapazes mais problemas externalizados (e.g., Zahn-Waxler, Shirtcliff \& Marceau, 2008). Em particular, o surgimento de sintomatologia internalizada é preocupante dado que o adolescente é o primeiro, e talvez o único, a dar-se conta do problema, pelo menos nas suas etapas iniciais.

Terceiro, na adolescência, os jovens passam menos tempo com os pais e têm cada vez mais autonomia na gestão das suas vidas (Coleman, 2011; Smetana, Robinson \& Rote, 2014), e assim a identificação de desajustamento psicológico e a iniciativa de pedir ajuda ficam também mais nas suas mãos.

Quarto, estudos sugerem resultados pouco otimistas relativamente à satisfação com a vida, um indicador positivo de saúde mental (Antaramian, Huebner, Hills \& Valois, 2010), dos adolescentes portugueses. Por exemplo, o estudo Health Behaviour in School-Aged Children (HBSC) (Inchley et 


\section{(DES)AJUSTAMENTO PSICOLÓGICO E EXPRESSÕES IDIOMÁTICAS NA ADOLESCÊNCIA}

al., 2015) mostrou que $26 \%$ das raparigas e $17 \%$ dos rapazes de 16 anos avaliam a sua satisfação com a vida com 5 ou menos pontos numa escala até 10 (Cantrill Ladder) (resultados semelhantes foram obtidos para adolescentes de 13 anos, e resultados um pouco mais otimistas para os 11 anos).

Quinto, de acordo com um estudo epidemiológico da saúde mental dos adultos portugueses, a prevalência de perturbações mentais é uma das mais altas entre os países estudados, $23 \%$ durante 0 ano anterior (Caldas de Almeida \& Xavier, 2013), reclamando assim alguma atenção para as trajetórias de (des)ajustamento desde a infância e adolescência. Como alguns estudos mostram, um dos melhores preditores da saúde mental na idade adulta é a saúde mental na adolescência (Chaiton et al., 2013; Reinherz, Paradis, Giaconia, Stashwick, \& Fitzmaurice, 2003).

\section{OBJETIVOS DA INVESTIGAÇÃO}

Os objetivos deste estudo são os de explorar, em adolescentes e considerando possíveis diferenças de género, a associação entre duas expressões idiomáticas relativas ao ajustamento psicológico comuns em Portugal, designadamente sofrer dos nervos (SN) e ter problemas pessoais (PP), e diversas dimensões de sintomatologia psicológica comuns na literatura científica da saúde mental. Os objetivos específicos são:

Descrever a autoperceção de (des)ajustamento psicológico com recurso às expressões idiomáticas SN e PP;

Descrever a gravidade da sintomatologia em 9 dimensões de psicopatologia;

Explorar a associação entre expressões idiomáticas e dimensões de psicopatologia;

Identificar preditores do (des)ajustamento referido através das expressões idiomáticas, entre as dimensões de psicopatologia.

Explorar diferenças de género, nos anteriores.

\section{MÉTODO}

\section{Participantes}

Participaram neste estudo 357 alunos do $12^{\circ}$ ano de escolaridade em três escolas secundárias do sul de Portugal, entre os quais 203 raparigas (56.86\%) e 154 rapazes (43.14\%). A média de idades é $17.50(\mathrm{DP}=1.05)$.

\section{Instrumentos}

0 Questionário de Dados Pessoais recolheu dados sobre variáveis sociodemográficas (idade, género, etc.) e sobre a autoperceção de (des)ajustamento psicológico. Neste caso, os adolescentes indicaram o seu nível de problemas usando a escala 0 - nada, 1 - pouco, ou 2 - muito, em dois itens de expressões idiomáticas, SN e PP, respetivamente.

0 Inventário de Sintomas Psicopatológicos (BSI) (Derogatis, 1982; Canavarro, 2007), que inclui 53 itens para classificar de 0 - nunca a 4 - muitíssimas vezes, mediu a gravidade da sintomatologia em 9 dimensões de psicopatologia: Somatização (Som), Obsessão-Compulsão (Ob-Comp), Sensibilidade Interpessoal (Sens Inter), Depressão (Depr), Ansiedade (Ans), Hostilidade (Hostil), Ansiedade Fóbica (Ans Fob), Ideação Paranóide (Id Paran), e Psicoticismo (Psicot). Esta versão apresenta boas qualidades psicométricas (ver Canavarro, 2007). A consistência interna obtida com a presente amostra é semelhante à obtida com a amostra da adaptação portuguesa, com Alphas de Cronbach desde .64 (Psicot) a .83 (Depr). 


\section{Procedimento}

Uma vez obtido o consentimento informado dos adolescents e/ou dos seus encarregados de educação, bem como dos diretores das escolas, os instrumentos foram administrados por psicólogas escolares durante uma aula, com o consentimento do(a) professor(a).

A análise dos dados realizou-se com ajuda do programa estatístico IBM SPSS 24. Foram analisadas as diferenças de género através de testes de Qui-Quadrado (para SN e PP) e testes t (diferença de médias nas dimensões do BSI). Foram calculadas correlações de Spearman para medir a associação entre dimensões de psicopatologia e as autoperceções de (des)ajustamento segundo as expressões idiomáticas, separadamente para cada género. Foram especialmente valorizadas as correlações com uma força pelo menos média, de acordo com as recomendações de Cohen (1988) (correlações superiores a .33).

Na busca de preditores para SN e PP, optou-se pela regressão logística para variáveis dicotómicas. Assim, SN e PP foram transformadas em duas variáveis dicotómicas, 0 - ausência de problemas e 1 - presença de problemas. Realizaram-se quatro análises de regressão logística, entrando SN ou PP como variável dependente e as 9 dimensões de psicopatologia do BSI como variáveis independentes, e usando o método forward stepwise (likelihood ratio), separadamente para cada género.

\section{RESULTADOS}

\section{Distribuição das Variáveis de SN e PP por Género}

Na tabela 1 mostra-se que um pouco mais de metade dos rapazes e das raparigas reportam um nível intermédio de SN e de PP, mas que há mais rapazes a reportar a ausência destes problemas e mais raparigas a reportar muitos problemas. Estas diferenças de género são significativas para SN, $\chi 2$ $(1,357)=12.850, p=.000, \phi=-.190$, mas não para PP, $\chi 2(1,355)=2.628, p=.105, \phi=-.086$.

Considerando os dois géneros separadamente, verifica-se que os rapazes reportam níveis mais elevados de desajustamento em PP do que em SN, McNemar test, $\chi^{2}=7.350, p=.007$, mas esta diferença não é significativa para as raparigas, McNemar test, $\chi^{2}=.845, p=.358$.

Tabela 1

Frequências das categorias de SN e de PP, por género (N, \%)

\begin{tabular}{|c|c|c|c|c|c|c|}
\hline \multirow{2}{*}{ Género } & \multicolumn{3}{|c|}{$\mathrm{SN}(N=357)$} & \multicolumn{3}{|c|}{$\mathrm{PP}(N=355)$} \\
\hline & Nada & Pouco & Muito & Nada & Pouco & Muito \\
\hline $\mathrm{F}$ & $54(26,6 \%)$ & $110(54,2 \%)$ & $39(19,2 \%)$ & $46(22,9 \%)$ & $127(63,2 \%)$ & $28(13,9 \%)$ \\
\hline M & $69(44,8 \%)$ & $79(51,3 \%)$ & $6(3,9 \%)$ & $47(30,5 \%)$ & $100(64,9 \%)$ & $7(4,5 \%)$ \\
\hline $\mathrm{F}+\mathrm{M}$ & $123(34,5 \%)$ & $189(52,9 \%)$ & $45(12,6 \%)$ & $93(26,2 \%)$ & $227(63,9 \%)$ & $35(9,9 \%)$ \\
\hline
\end{tabular}

Nota. F - género feminino; $\mathrm{M}$ - género masculino; $\mathrm{SN}$-sofrer dos nervos; $\mathrm{PP}$ - ter problemas pessoais.

\section{Estatísticas Descritivas das Dimensões de Psicopatologia do BSI e Diferenças de Género}

Os resultados da tabela 2 sugerem que as raparigas apresentam pontuações mais elevadas que os rapazes nas dimensões de Som, Sens Inter, Depr, Ans e Psicot. Não se verificaram diferenças de género nas dimensões de Ob-comp, Hostil, Ans Fob e Id Para. 
(DES)AJUSTAMENTO PSICOLÓGICO E EXPRESSÕES IDIOMÁTICAS NA ADOLESCÊNCIA

Tabela 2

Estatísticas descritivas (e teste $t$ ) das dimensões do BSI e correlações com SN e PP, por género

\begin{tabular}{|c|c|c|c|c|c|c|c|}
\hline \multirow{2}{*}{$\begin{array}{l}\text { Dimensões } \\
\text { psicopatologia (a) }\end{array}$} & \multicolumn{3}{|c|}{ Masculino } & \multicolumn{3}{|c|}{ Feminino } & \multirow{2}{*}{$\begin{array}{l}\text { Test } t \\
\text { (b) }\end{array}$} \\
\hline & $M(D P)$ & SN & PP & $M(D P)$ & SN & PP & \\
\hline SN & - & - & $.166^{*}$ & - & - & $.372^{* *}$ & - \\
\hline Som $^{*}$ &, $38(, 40)$ & .080 & $.213^{* *}$ &, $51(, 54)$ & $.322^{* *}$ & $.283^{* *}$ & -2.514 \\
\hline Obs-Com & $1,02(, 52)$ & $.214^{* *}$ & .140 & $1,11(, 60)$ & $.293^{* *}$ & $.309^{* *}$ & -1.493 \\
\hline Sens Inter ${ }^{* *}$ &, $74(, 57)$ & $.166^{*}$ & $.355^{* *}$ &, $99(, 71)$ & $.295^{* *}$ & $.182^{* *}$ & -3.631 \\
\hline $\operatorname{Depr}^{* *}$ &, $68(, 67)$ & $.201^{*}$ & $.397^{* *}$ & ,95 (,64) & $.302^{* *}$ & $.366^{* *}$ & -3.860 \\
\hline Ans ${ }^{* *}$ &, $54(, 47)$ & $.353^{* *}$ & $.231^{* *}$ &, $85(, 66)$ & $.461^{* *}$ & $.315^{* *}$ & -5.152 \\
\hline Hostil &, $84(, 63)$ & $.163^{*}$ & $.223^{* *}$ & ,93 $(, 70)$ & $.223^{* *}$ & $.295^{* *}$ & -1.306 \\
\hline Ans Fób & ,29(,41) & .118 & $.239^{* *}$ &, $34(, 45)$ & $.321^{* *}$ & $.183^{* *}$ & -1.102 \\
\hline Id Paran & $1,09(, 65)$ & $.193^{*}$ & $.372^{* *}$ & $1,02(, 65)$ & $.288^{* *}$ & $.351^{* *}$ & 1.120 \\
\hline Psicot ${ }^{*}$ &, $60(, 54)$ & $.214^{* *}$ & $.420^{* *}$ &, $74(, 62)$ & $.288^{* *}$ & $.322^{* *}$ & -2.369 \\
\hline
\end{tabular}

Nota. SN - sofrer dos nervos; PP - ter problemas pessoais; Som - somatização; Obs-Com - obsessão-compulsão; Sens Inter - sensibilidade interpessoal; Depr - depressão; Ans - ansiedade; Hostil - hostilidade; Ans Fob ansiedade fóbica; Id Paran - ideação paranoide; Psicot - psicoticismo.

(a) Nesta coluna assinalam-se com asterisco(s) as diferenças de género estatisticamente significativas (teste $t$ ).

(b) Graus de liberdade $=355$, exceto para Sens Interp (=353.817) e Ans (=354.160).

** $p<.01 ; * p<.05$

\section{Correlações entre Expressões Idiomáticas de Desajustamento e Dimensões de Psicopatologia do BSI}

A tabela 2 mostra que as autoperceções de SN e de PP estão significativamente correlacionadas, sendo esta correlação mais forte no género feminino.

Além disso, todas as correlações entre expressões idiomáticas de desajustamento e dimensões de psicopatologia são estatisticamente significativas no género feminino. Encontra-se um cenário idêntico para o género masculino, com a exceção das correlações não significativas entre SN e Som ou Ans Fob, e entre PP e Ob-Comp.

Atendendo à força das correlações ( $r>$.33), verifica-se que, para ambos os géneros, a correlação mais forte de SN é com Ans. As correlações mais fortes de PP ocorrem com Psicot, Depr, Id Paran e Sens Inter, no género masculino, e, no género feminino, com Depr e Id Paran.

\section{Dimensões de Psicopatologia como Preditoras das Expressões Idiomáticas de Desajustamento}

A tabela 3 sintetiza as estatísticas dos 4 modelos de regressão logística e dos preditores de SN e de PP que se afiguraram, para rapazes e para raparigas. Todos os modelos se completaram após o primeiro passo, todos revelaram descrever melhor os dados do que um modelo sem preditores (teste dos coeficientes do modelo) e adequar-se bem aos dados (teste de Hosmer \& Lemeshow); a única exceção vai para o modelo relativo a SN nas raparigas, que não se ajusta tão bem aos dados, modelo que assim se considerará com algumas reservas. Em cada um dos modelos testados só uma dimensão de psicopatologia emerge como preditor estatisticamente significativo.

A Ans apresenta-se como o melhor preditor de SN para ambos os géneros, e os modelos com este preditor permitem classificar corretamente $65 \%$ dos rapazes nas duas categorias da variável, e $80 \%$ das raparigas (com as reservas mencionadas acima), respetivamente.

0 melhor preditor de PP, para o género masculino, é Psicot, e permite classificar corretamente $73 \%$ dos rapazes nas duas categorias de PP. Já o melhor preditor de PP para 0 género feminino é a Id Paran, permitindo o modelo classificar $77.1 \%$ das raparigas de acordo com 0 seu reporte de ter ou não PP. 
Tabela 3

Modelos de regressão logística (síntese)

\begin{tabular}{|c|c|c|c|c|c|c|c|c|}
\hline \multirow{2}{*}{$\begin{array}{l}\text { V. } \\
\text { Dep }\end{array}$} & \multirow[t]{2}{*}{ Género } & \multirow[t]{2}{*}{ Preditor } & \multirow[t]{2}{*}{$B$} & \multirow[t]{2}{*}{ Wald } & \multirow{2}{*}{$\begin{array}{l}\operatorname{Exp} \\
\text { (B) }\end{array}$} & \multicolumn{2}{|c|}{ Testes do Modelo } & \multirow{2}{*}{$\begin{array}{c}R^{2} \\
\text { Nagelkerke }\end{array}$} \\
\hline & & & & & & $\begin{array}{c}\text { Teste dos } \\
\text { coeficientes }\end{array}$ & $\begin{array}{l}\text { Hosmer \& } \\
\text { Lemeshow }\end{array}$ & \\
\hline SN & $\mathrm{M}$ & Ans & 1.909 & $\begin{array}{l}\text { Qui }^{2}(1)= \\
15.169, p= \\
.000\end{array}$ & 6.749 & $\begin{array}{c}\operatorname{Qui}^{2}(1,154)= \\
20.350, p= \\
.000\end{array}$ & $\begin{array}{l}\text { Qui }^{2}(6,154)= \\
3.057, p=.802\end{array}$ & 0.166 \\
\hline SN & $\mathrm{F}$ & Ans & 2.027 & $\begin{array}{l}\mathrm{Qui}^{2}(1)= \\
22.231, p= \\
.000\end{array}$ & 7.595 & $\begin{array}{c}\operatorname{Qui}^{2}(1,203)= \\
33.903, p= \\
.000\end{array}$ & $\begin{array}{c}\mathrm{Qui}^{2}(8,203)= \\
16.407, p= \\
.037\end{array}$ & 0.224 \\
\hline PP & M & Psicot & 2.283 & $\begin{array}{l}\mathrm{Qui}^{2}(1)= \\
17.255, p= \\
.000\end{array}$ & 9.803 & $\begin{array}{c}\operatorname{Qui}^{2}(1,154)= \\
25.975, p= \\
.000\end{array}$ & $\begin{array}{l}\operatorname{Qui}^{2}(5,154)= \\
2.725, p=.742\end{array}$ & 0.219 \\
\hline PP & $\mathrm{F}$ & $\begin{array}{c}\text { Id } \\
\text { Paran }\end{array}$ & 1.321 & $\begin{array}{l}\operatorname{Qui}^{2}(1)= \\
13.298, p= \\
.000\end{array}$ & 3.748 & $\begin{array}{c}\operatorname{Qui}^{2}(1,203)= \\
17.202, p= \\
.000\end{array}$ & $\begin{array}{c}\operatorname{Qui}^{2}(7,203)= \\
12.765, p= \\
.078\end{array}$ & 0.124 \\
\hline
\end{tabular}

Nota: V. Dep - variável dependente; SN - sofrer dos nervos; PP - ter problemas pessoais; M - género masculino; F - género feminino; Ans - ansiedade; Psicot - psicoticismo; Id Paran - ideação paranoide.

\section{DISCUSSÃO}

Os objetivos deste estudo eram os de descrever a distribuição da autoperceção de desajustamento por meio de expressões idiomaticas e a distribuição de sintomas em diferentes dimensões de psicopatologia e, sobretudo, explorar a associação entre ambas os relatos de desajustamento psicológico, procurando identificar diferenças de género.

Os resultados mostraram que há diferenças de género no reportar de (des)ajustamento psicológico por meio de expressões idiomáticas como SN, reportando as raparigas mais problemas que os rapazes, o que vai ao encontro de alguma literatura existente (e.g., James et al., 2009). No entanto, não se verificam diferenças de género quando se trata de avaliar a presença de PP. Uma hipótese é a de que esta expressão idiomática seja socialmente percebida de modo mais benigno, ou seja reservada a problemas transitórios mesmo que graves, e assim os rapazes mais facilmente a refeririam. De facto, no seu estudo, Alves (2011), encontrou dois tipos de significações para as expressões idiomáticas relacionadas com saúde e doença mental. Um desses tipos refere-se a sofrimento mental visto este como normal (como uma reação a um acontecimento) ou continuado (percebido como parte da personalidade, e que pode associar-se a uma percepção cultural de fragilidade da pessoa), ligado a expressões idiomáticas como sofrer dos nervos ou cismas. 0 outro tipo refere-se a condições vistas como defeito (e não como fragilidade) da pessoa e que assim afeta a sua identidade e a diminui perante a sociedade; incluem-se aqui expressões como "doença da cabeça" ou "doença dos nervos". A assumpção de sofrer muito dos nervos implicaria sempre, neste quadro, alguma desvalorização perante si mesmo e a sociedade, o que poderá acontecer de modo diferente com PP. Mais estudos são necessários para compreender todo o significado atribuído a estas expressões idiomáticas e a outras que eventualmente sejam frequentes entre os jovens. De facto neste estudo os rapazes reportam níveis mais elevados de PP que de SN e não se distinguem das raparigas no reportar de PP.

Por outro lado, tal como em SN as raparigas reportaram níveis mais elevados em diferentes dimensões da sintomatologia internalizada, como Ans, Depr, Sens Inter, Som e Psicot, indo ao encontro dos resultados de investigação anterior (e.g., Hankin, 2015; Zahn-Waxler et al., 2008). Por outro lado, em dimensões mais cognitivas da sintomatologia (e.g., Ob-Comp, Id Paran) ou afetos 


\section{(DES)AJUSTAMENTO PSICOLÓGICO E EXPRESSÕES IDIOMÁTICAS NA ADOLESCÊNCIA}

orientados mais para o exterior (Host, Ans Fob) não se verificaram diferenças entre rapazes e raparigas.

Finalmente, ambas as expressões idiomáticas de desajustamento psicológico apareceram associadas à generalidade das dimensões de psicopatologia do BSI (especialmente PP) em ambos os géneros. No entanto, as correlações mais fortes ligaram SN à Ans e esta afigurou-se também 0 melhor preditor de SN, tanto para os rapazes como para as raparigas. Este resultado apoia James e colegas (2009) quando referem que as descrições de sofrer dos nervos cumprem os critérios de diagnóstico de perturbação de ansiedade generalizada, perturbação de pânico, e/ou perturbação de stresse pós-traumático, embora no presente estudo não seja tão forte a sua ligação a diagnósticos de depressão e distimia, como os autores também referem.

Já o padrão de associação de PP com as dimensões de psicopatologia parece ser diferente em função do género. Embora PP apareça fortemente associação com Dep e Id Paran em ambos géneros, no género masculino PP também se correlaciona com Sens Inter e Psicot. Assim, no género masculino o melhor preditor de PP é Psicot (e.g., isolamento e evitamento social, dificuldades no controlo do pensamento) e no género feminino Id Paran (ex., um modo de funcionamento cognitivo caraterizado por pensamento projetivo, suspeição, etc.). Portanto, no género masculino, mais que no feminino, PP parece associar-se a dificuldades nas relações interpessoais. Mais estudos são necessários para aprofundar as relações encontradas.

\section{CONCLUSÕES}

Este estudo é um contributo para esclarecer a associação entre 0 uso de expressões idiomáticas sobre 0 desajustamento psicológico em adolescentes e diversas dimensões da psicopatologia correntes na linguagem técnico-científica da área da saúde mental.

Os resultados obtidos relativamente a SN são consistentes com alguma literatura existente, mas a compreensão da significação de PP mostrou-se mais desafiante: sem diferenças de género no reporte destes problemas de ajustamento, as diferenças revelam-se nos padrões diferentes de associação com as dimensões de psicopatologia e no emergir de diferentes preditores para cada género.

Mais estudos são necessários para esclarecer a significação que os adolescentes atribuem a estas expressões idiomáticas de desajustamento psicológico (e a outras porventura mais usuais entre os jovens portugueses) para que possam também beneficiar a comunicação entre adolescentes e profissionais de saúde mental.

\section{REFERÊNCIAS BIBLIOGRÁFICAS}

Abreu, J. L. P. (2007). Como Tornar-se Doente Mental (17th Ed.). Lisboa, Portugal: D. Quixote.

Alves, F. (2011). A doença mental nem sempre é doença: racionalidades leigas sobre saúde e doença mental. Porto, Portugal: Afrontamento.

American Psychiatric Association (2013). Diagnostic and Statistical Manual of Mental Disorders (5 ${ }^{\text {th }}$ Ed.). Washington D.C.: A.P.A.

Antaramian, S. P., Huebner, E. S., Hills, K. J., \& Valois, R. F. (2010). A dual factor model of mental health: Toward a more comprehensive understanding of youth functioning. American Journal of Orthopsychiatry, 80(4), 462.

Arnett, J.J. (1999). Adolescent Storm and Stress, Reconsidered. American Psychologist, vol.54, ํㅡㄴ $5,317-326$.

Caldas de Almeida, J. \& Xavier, M., (Coord) (2013). Estudo Epidemiológico Nacional de Saúde Mental. 1. Relatório. Lisboa: Faculdade de Ciências Médicas, Universidade Nova de Lisboa. Available in: http://www. fcm. unl. pt/main/alldoc/galeria_imagens/Relatorio_Estudo_SaudeMental_2.pdf. 
Canavarro, M. C. (2007). Inventario de Sintomas Psicopatologicos: Uma Revisao critica dos estudos realizados em Portugal. In L. Almeida, M. Simoes, C. Machado e M. Goncalves (Eds.), Avaliacao psicologica. Instrumentos validados para a populacao portuguesa, vol. III (pp. 305331). Coimbra: Quarteto Editora.

Carapeto, M. J. \& Feixas, G. (2019). Self-knowledge and depressive symptoms in late adolescence: A study using the repertory grid technique. Journal of Constructivist Psychology, 32:1, 81-97. https://doi.org/10.1080/10720537.2018.1433087

Causadias, J. M. (2013). A roadmap for the integration of culture into developmental psychopathology. Development and Psychopathology, 25(4pt2), 1375-1398.

Chaiton, Contreras, Brunet, Sabiston, O'Loughlin, Low, ... \& O'Loughlin (2013). Heterogeneity of depressive symptom trajectories through adolescence: predicting outcomes in young adulthood. Journal of the Canadian Academy of Child and Adolescent Psychiatry, 22(2), 96.

Cicchetti, D., \& Rogosch, F.A. (2002). Developmental Psychopathology Perspective on Adolescence. Journal of Consulting and Clinical Psychology, 70 (1), 6-20.

Cohen, J. (1988). Statistical power analysis for the behavioral sciences (2nd ed.). Hillsdale, NJ: Erlbaum.

Coleman, J. C. (2011). The nature of adolescence (4th Ed). New York, NY: Psychology Press.

Derogatis, L. (1982). BSI: Brief symptom inventory. Minneapolis. MN: National Computers Systems.

Garber, J., Weersing, V. R., Hollon, S. D., Porta, G., Clarke, G. N., Dickerson, J. F., ... \& Brent, D. A. (2018). Prevention of depression in at-risk adolescents: moderators of long-term response. Prevention Science, 19(1), 6-15.

Hankin, B. L. (2015). Depression from childhood through adolescence: risk mechanisms across multiple systems and levels of analysis. Current Opinion in Psychology, 4, 13-20. http://dx.doi.org/10.1016/j.copsyc.2015.01.003

Inchley, J., \& Currie, D. (2016). Growing up unequal: gender and socioeconomic differences in young people's health and well-being. Health Behaviour in School-aged Children (HBSC) study: international report from the 2013/2014 survey. Copenhagen, Denmark: WHO Regional Officece for Europe.

James, S., Fernandes, M., Navara, G. S., Harris, S., \& Foster, D. (2009). Problemas de nervos: A multivocal symbol of distress for Portuguese immigrants. Transcultural psychiatry, 46(2), 285299.

James, S., Slocum, S. L., \& Zumbo, B. D. (2004). Measuring agonias among female Portuguese immigrants: construct description and psychometric properties. International Journal of Testing, 4(2), 183-188.

Kaiser, B. N., Haroz, E. E., Kohrt, B. A., Bolton, P. A., Bass, J. K., \& Hinton, D. E. (2015). "Thinking too much": a systematic review of a common idiom of distress. Social Science \& Medicine, 147, 170-183.

Nogueira, B. L., Mari, J. D. J., \& Razzouk, D. (2015). Culture-bound syndromes in Spanish speaking Latin America: the case of Nervios, Susto and Ataques de Nervios. Archives of Clinical Psychiatry (São Paulo), 42(6), 171-178.

Reinherz, H. Z., Paradis, A. D., Giaconia, R. M., Stashwick, C. K., \& Fitzmaurice, G. (2003). Childhood and adolescent predictors of major depression in the transition to adulthood. American Journal of Psychiatry, 160(12), 2141-2147.

Smetana, J. G., Robinson, J., \& Rote, W. M. (2014). Socialization in adolescence. In J. E. Grusec and P. D. Hastings (Eds.), Handbook of Socialization: Theory and Research (2 ${ }^{\text {nd }}$ Ed.) (pp. 60-84). Guilford. 


\section{(DES)AJUSTAMENTO PSICOLÓGICO E EXPRESSÕES IDIOMÁTICAS NA ADOLESCÊNCIA}

World Health Organization (1992). International statistical classification of disease and related health problems, Tenth Revision (ICD-10). Geneva: World Health Organization.

Zahn-Waxler, C., Shirtcliff, E. A., \& Marceau, K. (2008). Disorders of childhood and adolescence: Gender and psychopathology. Annual Review of. Clinical Psychology, 4, 275-303. https://doi.org/10.1146/annurev.clinpsy.3.022806.091358 